\title{
Pathogenesis of Renal Injury in the Megabladder Mouse: A Genetic Model of Congenital Obstructive Nephropathy
}

\author{
SUSAN E. INGRAHAM, MONALEE SAHA, ASHLEY R. CARPENTER, MELISSA ROBINSON, IHAB ISMAIL, SUNITA SINGH, \\ DAVID HAINS, MICHAEL L. ROBINSON, DANIEL A. HIRSELJ, STEPHEN A. KOFF, CARLTON M. BATES, \\ AND KIRK M. MCHUGH

\begin{abstract}
Center for Molecular and Human Genetics [S.E.I., M.S., A.R.C., M.R., I.I., S.S., K.M.M.], Sections of Nephrology [S.E.I., D.H., K.M.M.] and Urology [D.A.H., S.A.K.], Nationwide Children's Hospital, Columbus, Ohio 43205; Department of Zoology [M.L.R.], Miami

University, Oxford, Ohio 45056; Section of Nephrology [C.M.B.], Children's Hospital of Pittsburgh, Pittsburgh, Pennsylvania 15201
\end{abstract}

\begin{abstract}
Congenital obstructive nephropathy (CON) is the most common cause of chronic renal failure in children often leading to end-stage renal disease. The megabladder $(m g b)$ mouse exhibits signs of urinary tract obstruction in utero resulting in the development of hydroureteronephrosis and progressive renal failure after birth. This study examined the development of progressive renal injury in homozygous $m g b$ mice $(m g b-/-)$. Renal ultrasound was used to stratify the disease state of $m g b-/-$ mice, whereas surgical rescue was performed using vesicostomy. The progression of renal injury was characterized using a series of pathogenic markers including alpha smooth muscle isoactin ( $\alpha$-SMA), TGF- $\beta 1$, connective tissue growth factor (CTGF), E-cadherin, F4/80, Wilm's tumor (WT)-1, and paired box gene (Pax) 2. This analysis indicated that $m g b-/-$ mice are born with pathologic changes in kidney development that progressively worsen in direct correlation with the severity of hydronephrosis. The initiation and pattern of fibrotic development observed in mgb-/- kidneys appeared distinctive from previous animal models of obstruction. These observations suggest that the $m g b$ mouse represents a unique small animal model for the study of CON. (Pediatr Res 68: 500-507, 2010)
\end{abstract}

$\mathrm{C}$ ongenital obstructive nephropathy (CON) is a complex disease involving pathologic changes in kidney development and function that occur as a result of obstructed urine flow in utero. CON is the most common cause of chronic renal failure in children (1). Its clinical course and outcome are dependent on the developmental stage at which the obstruction occurs, the degree and duration of the obstruction, and whether one or both kidneys are involved. Although surgical intervention can relieve some effects of CON, many pathologic changes associated with this condition seem irreversible (2). Diagnostic, therapeutic, and prognostic quandaries often arise in the management of CON, creating a significant need for animal models of this important disease.

We recently identified a unique mutant mouse designated $m g b$ for megabladder. These mice develop signs of lower urinary tract obstruction in utero secondary to a nonfunctional, over-distended bladder, with signs of renal failure evident

Received April 21, 2010; accepted July 17, 2010

Correspondence: Kirk M. McHugh, Ph.D., The Research Institute at Nationwide Children's Hospital, 700 Children's Drive, Columbus, OH 43205; e-mail: kirk.mchugh@nationwidechildrens.org

Supported by NIH R01-DK70907, R01-DK085242, and R01-EY12995.

S.E.I. and M.S. contributed equally to this study. shortly after birth (3). Male homozygotes ( $m g b-/-)$ develop early renal insufficiency and rarely survive beyond $4-6 \mathrm{wk}$, whereas female $m g b-/-$ mice may live up to a year (3). The aim of this study was to assess and compare the progression of renal disease in $m g b-/-$ mice with previous experimental and genetic models of obstruction as well as human CON.

\section{METHODS}

Mice. Animals were maintained according to National Institutes of Health Guide for the Care and Use of Laboratory Animals with approval from the Institutional Animal Care and Use Committee of Nationwide Children's Hospital. Developmental stages included embryonic d 15.5 (E15.5), E17.5, newborn (NB, postnatal d 0-3), and adult (AD, 3 wk or older). Controls were age-matched $\mathrm{FVB} / \mathrm{N}$ mice. Genotyping was performed as previously described (3).

Histological analysis. E15.5 embryos and individual kidneys were fixed in $10 \%$ formalin. Masson's trichrome was performed using Sigma Chemical Co.-Aldrich Trichrome kit (St. Louis, MO). Hematoxylin and eosin staining was performed using standard procedures. Certified veterinary pathologists at Vet Path Services, Inc. (Mason, OH) or The Mouse Phenotyping Core (The Ohio State University, Columbus, $\mathrm{OH}$ ) performed histopathological assessments. Tissues were processed in Leica TP 1050 Automatic Tissue Processor (Leica Microsystems, Wetzlar, Germany) and stained with antibodies for alpha smooth muscle isoactin ( $\alpha$-SMA; DakoCytomation, Carpinteria, CA); F4/80 (AbD Serotec, Raleigh NC); aquaporin (AQP) 2; TGF- $\beta 1$; connective tissue growth factor (CTGF); Wilm's tumor (WT)-1 (Santa Cruz Biotechnology, Santa Cruz, CA); E-cadherin (Cell Signaling, Danvers, MA); and paired box gene (Pax2, Invitrogen, Camarillo, CA) as outlined by suppliers. TUNEL was performed according to manufacturer's instructions (CalBioChem, Gibbstown, NJ).

The 3-dimensional (3D) reconstruction and quantitation were performed using StereoInvestigator and Neurolucida Explorer (MBF Bioscience, Williston, VT). Section intervals were set to desired $\mathrm{z}$ depth and outer limits of each structure traced and enclosed at optimal magnification. Sections were aligned, stacked, and connected using 3D wireframe view, quantitated, and compared by $t$ test. A $50-\mu \mathrm{m}$ grid was overlaid on each $10 \times$ image and fibrosis graded as less than or greater than $50 \%$ positive staining. Total percentage of grid squares with $>50 \%$ positive staining was calculated by software. Positively staining glomerular cells were counted by hand. Results were compared by $t$ test.

Abbreviations: $\boldsymbol{\alpha}$-SMA, alpha smooth muscle isoactin; AD, adult; APD, anterior posterior diameter; AQP2, aquaporin-2; CON, congenital obstructive nephropathy; CTGF, connective tissue growth factor; E, embryonic day; EMT, epithelial-to-mesenchymal transition; $\boldsymbol{m g} \boldsymbol{b}$, megabladder; NB, newborn; Pax2, paired box gene 2; RL, renal length; UUO, unilateral ureteral obstruction; WT-1, Wilm's tumor 1 
Renal ultrasound. Renal ultrasound was performed on 2, 4, and 5-wk male mice using VisualSonics In Vivo Model RMV-704 and $40 \mathrm{MHz}$ small animal probe (Toronto, ON). Total renal length (RL) and renal pelvic anteroposterior diameter (APD) were measured. Hydronephrosis was scored by the ratio of APD to RL. Individual kidneys were classified as no hydronephrosis (APD/ $\mathrm{RL}=0$ ), mild hydronephrosis (APD/RL $>1$ SD below mean), moderate hydronephrosis $(\mathrm{APD} / \mathrm{RL}=$ mean $\pm 1 \mathrm{SD})$, or severe hydronephrosis (APD/RL $>1$ SD above mean).

Vesicostomy. Four-week old male $m g b-/-$ mice underwent vesicostomy using inhalation anesthesia. Abdominal fur was removed, and a $15-\mathrm{mm}$ low transverse abdominal incision was made lateral to midline. Peritoneum was divided transversely and bladder brought into the surgical field. A 24-gauge angiocatheter was introduced into the bladder, and contents were aspirated. After catheter removal, cystotomy was enlarged, and four-quadrant fixation of bladder edges to peritoneum and abdominal wall was performed with interrupted 6-0 polydioxanone sutures. Additional sutures were added for circumferential closure. Antibiotic ointment was applied and stomal patency monitored.

\section{RESULTS}

Disease stratification. Renal injury was stratified in $m g b-/-$ mice using renal ultrasound (Fig. 1). Eighteen $m g b-/-$ mice and 19 age-matched controls were studied at 2, 4 , and 5 wk of age. No difference in RL was observed between control and $m g b-/-$ mice at any time point. Hydronephrosis was not observed in any control kidneys. Hydronephrosis in $m g b-1-$ animals progressively worsened during the time course of the study; 15 of $18 \mathrm{mgb}-/-$ mice developed bilateral hydronephrosis; and the remaining three developed unilateral hydronephrosis. Renal pelvic APD was significantly increased at all time points with a majority of right kidneys showing greater hydronephrosis than left at each time point (Table 1). The difference in degree of left APD between 5 -wk-old survivors and nonsurvivors was statistically significant $(p<0.05)$, whereas the degree of left kidney preservation seemed predictive of earlier demise.

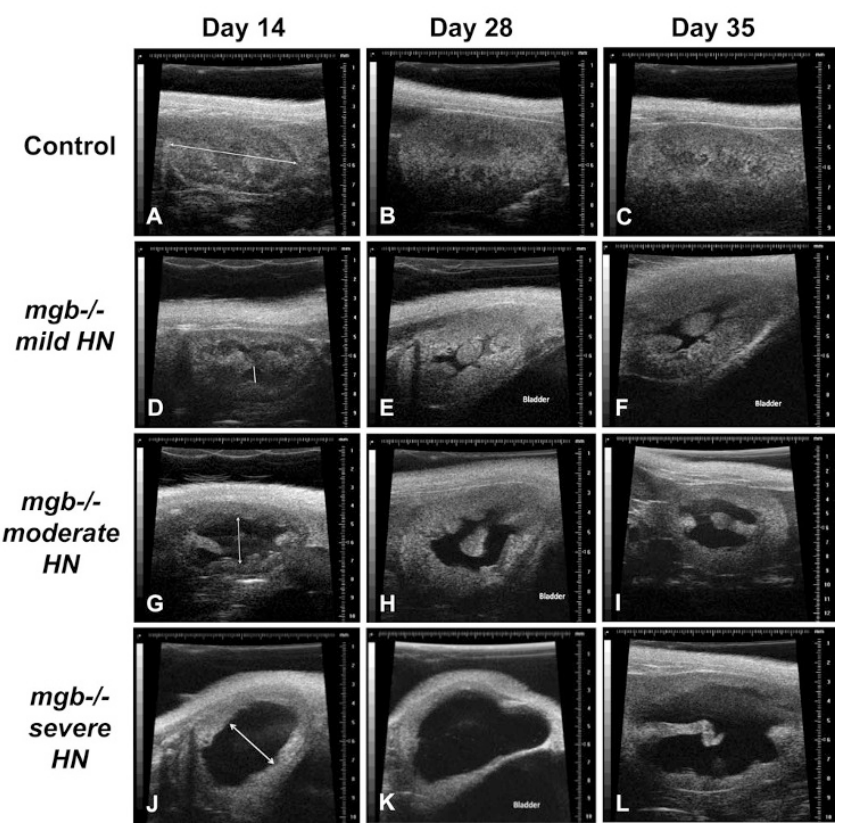

Figure 1. Renal ultrasound at 14,28 , and 35 d postnatal. Kidneys from control $(A-C)$ and $m g b-/-$ mice that show mild $(D-F)$, moderate $(G-I)$, and severe $(J-L)$ hydronephrosis $(\mathrm{HN})$. Line in panel $A$ indicates RL. Doubleheaded arrow in panels $D, G$, and $J$ indicates renal pelvis APD
Table 1. Mean renal pelvic APD ultrasound measurements in control and $\mathrm{mgb}-1-$ mice at 14,28 , and $35 \mathrm{~d}$

\begin{tabular}{clccc}
\hline & \multicolumn{3}{c}{ Mean APD $(\mathrm{mm})$} & \\
\cline { 2 - 4 } Age & Kidney & Control & $m g b-/-$ & \multicolumn{1}{c}{$p$} \\
\hline D 14 & Right & 0 & 1.59 & 0.0001 \\
& Left & 0 & 0.71 & 0.011 \\
D 28 & Right & 0 & 1.97 & 0.007 \\
& Left & 0 & 0.63 & 0.04 \\
D 35 & Right & 0 & 2.29 & 0.002 \\
& Left & 0 & 0.72 & 0.03 \\
\hline
\end{tabular}

Table 2. Total kidney volume vs renal pelvic volume as determined by three-dimensional reconstruction of E17.5 kidneys

\begin{tabular}{ccccc}
\hline & $m g b-/-$ Right & $m g b-/-$ Left & Control right & Control left \\
\hline $\begin{array}{c}\text { Total kidney } \\
\text { volume } \\
\left(\mu \mathrm{m}^{3}\right)\end{array}$ & $2.10 \times 10^{9}$ & $1.85 \times 10^{9}$ & $1.34 \times 10^{9}$ & $1.29 \times 10^{9}$ \\
$\begin{array}{c}\text { Renal pelvis } \\
\text { volume } \\
\left(\mu \mathrm{m}^{3}\right)\end{array}$ & $8.67 \times 10^{7}$ & $6.27 \times 10^{7}$ & $1.32 \times 10^{7}$ & $1.31 \times 10^{7}$ \\
$\begin{array}{c}\mathrm{RPV} \text { as } \% \\
\text { of TKV* }\end{array}$ & $4.1^{*}$ & $3.4^{*}$ & 1.0 & 1.0 \\
\hline
\end{tabular}

$* P=0.018$.

E15.5 and E17.5 kidneys. No differences in expression were observed at E15.5 for any of the histopathological markers examined (data not shown). E17.5 $\mathrm{mgb}-/-$ mice showed the first signs of megabladder, lower urinary tract obstruction, and hydroureteronephrosis that resulted in a significant increase in renal pelvis volume (Table 2). Nevertheless, E17.5 mgb-/- kidneys showed no pathogenic changes in any of the markers examined (data not shown).

NB kidneys. NB $m g b-/-$ mice typically showed mild to moderate unilateral hydroureteronephrosis and possessed mild, diffuse vacuolation of renal tubule epithelium and expanded cortical and medullary $\alpha$-SMA staining versus controls (Fig. 2). These changes were seen in both the hydronephrotic and contralateral kidneys. No differences were observed for any of the remaining biomarkers examined (data not shown).

AD kidneys. Histopathological review of 12-paired AD $m g b-/-$ kidneys confirmed the presence of unilateral hydronephrosis in the right kidney of nine animals and bilateral hydronephrosis in the remaining three. Moderate to severe kidneys showed minimal to marked loss of renal parenchyma accompanied by both tubular and glomerular atrophy. Severe kidneys showed moderate to severe interstitial fibrosis with varying degrees of medullary and subcapsular fibrosis. Simple cysts and cystic dilation of renal tubules with proteinaceous casts were also observed (Fig. $3 A-C$ ). Glomeruli within these severe kidneys ranged from globally sclerotic to mildly hypercellular with occasional neutrophils (Fig. 3D). Multifocal coagulation necrosis and vacuolization of tubular epithelial cells were also observed (Fig. 3E). Severe kidneys showed varying degrees of inflammation (Fig. 4), including microabscesses and chronic interstitial inflammatory infiltrates consisting of mononuclear leukocytes and plasma cells, some of which contained Russell bodies.

Collagen deposition in $\mathrm{AD} m g b-/-$ kidneys initiated just below the urothelium of the renal pelvis followed by progres- 


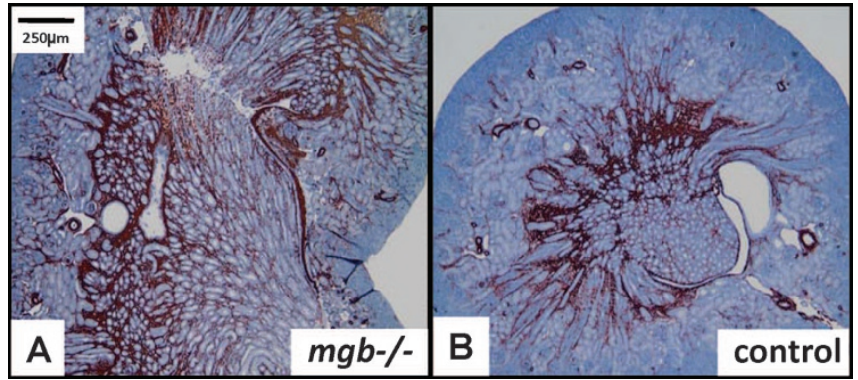

Figure 2. Expanded cortical and medullary $\alpha$-SMA expression in NB $m g b-/-$ kidney $(A) v s \mathrm{NB}$ control $(B), \times 5$.

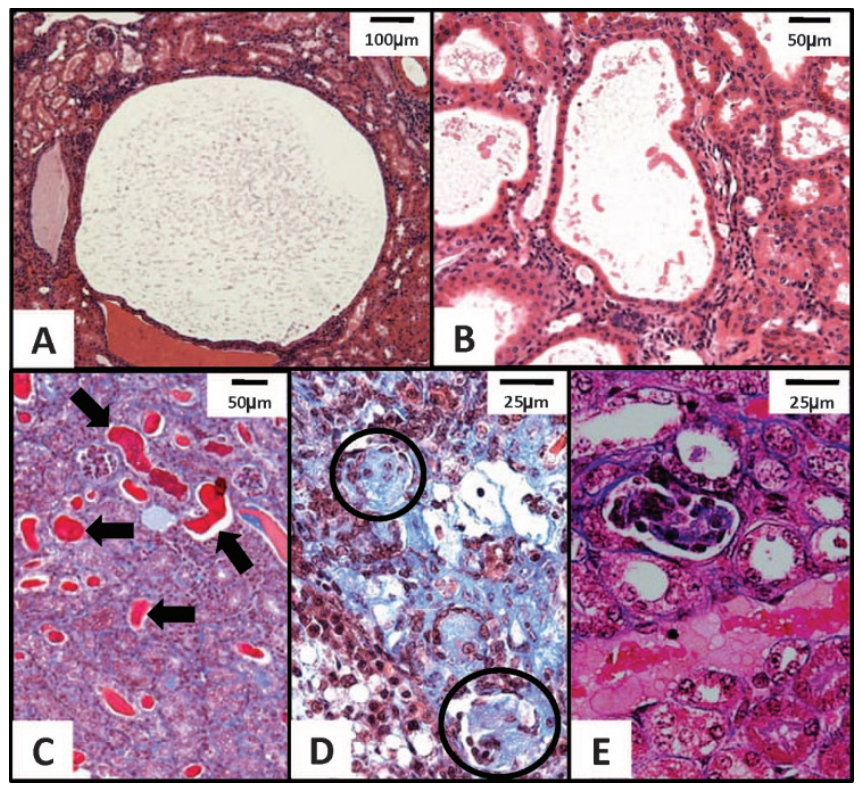

Figure 3. Histopathological features in $\mathrm{AD} m g b-/-$ kidneys. H\&E staining of $(A)$ simple cyst in renal cortex in severe kidney, $\times 10 ;(B)$ cystic dilation of renal tubules in severe kidney, $\times 20$. Trichrome of $(C)$ moderate kidney cortex with mildly to severely (arrows) dilated tubules containing numerous proteinaceous casts, $\times 20$; $(D)$ globally sclerotic glomeruli (circled) in severe kidney, $\times 40 ;(E)$ coagulation necrosis and vacuolization of tubular epithelium in moderate kidney, $\times 40$.

sive involvement of the subcapsular tissue and renal interstitium (Fig. 5). Interestingly, collagen deposition underlying the urothelium of the renal pelvis was also observed in the contralateral nonhydronephrotic kidney (Fig. 5B). Severe AD kidneys showed a statistically significant increase in collagen deposition in both the cortex and medulla (Fig. 6).

AD $m g b-/-$ kidneys showed more widespread $\alpha$-SMA expression than controls (Fig. 7A and $B$ ). Severe kidneys showed intense $\alpha$-SMA expression under the urothelium of the renal pelvis, underlying the renal capsule, and throughout the interstitium (Fig. 7C-E). Interstitial $\alpha$-SMA-positive cells were predominantly observed within the peritubular space and often formed smooth muscle collars, whereas scattered $\alpha$-SMApositive tubular cells were also observed (Fig. $7 F$ and $G$ ).

Moderate kidneys showed more intense TGF- $\beta 1$ expression within medullary tubules, cortical and medullary interstitial cells, and glomeruli compared with controls (Fig. 8A-F). Severe kidneys showed expanded TGF- $\beta 1$ staining in glomeruli, interstitial cells, capsular epithelium, urothelium of the

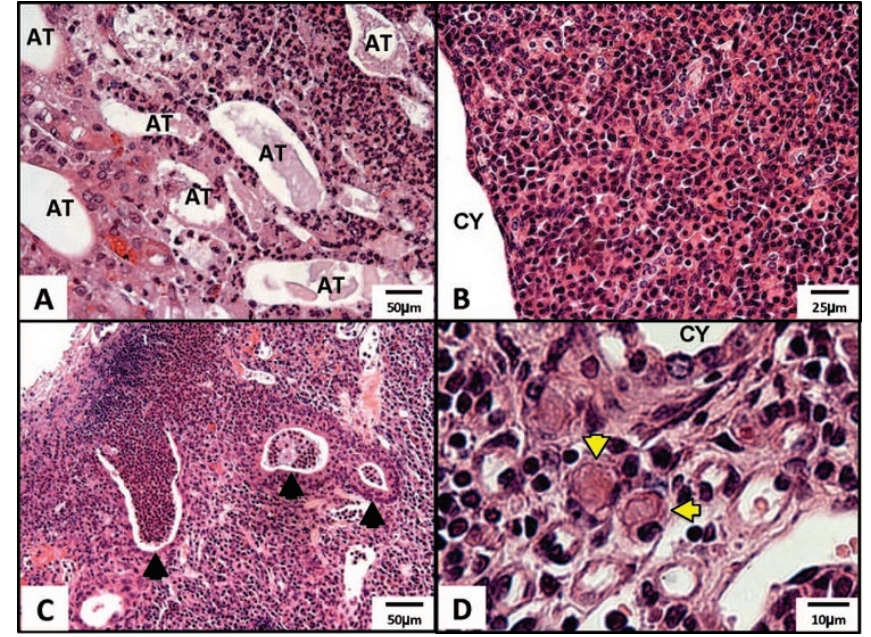

Figure 4. Inflammation in severe AD $m g b-/-$ kidneys. H\&E staining of (A) renal papilla showing atrophic, dilated tubules $(A T)$ surrounded by inflammatory infiltrate, $\times 20 ;(B)$ inflammatory infiltrate in the cortex adjacent to cystic dilation $(C Y), \times 40 ;(C)$ abscesses (black arrowheads) in the outer cortex, $\times 20$; and $(D)$ inflammatory infiltrate in cortex showing plasma cells including Russell bodies (yellow arrowheads), $\times 100$.

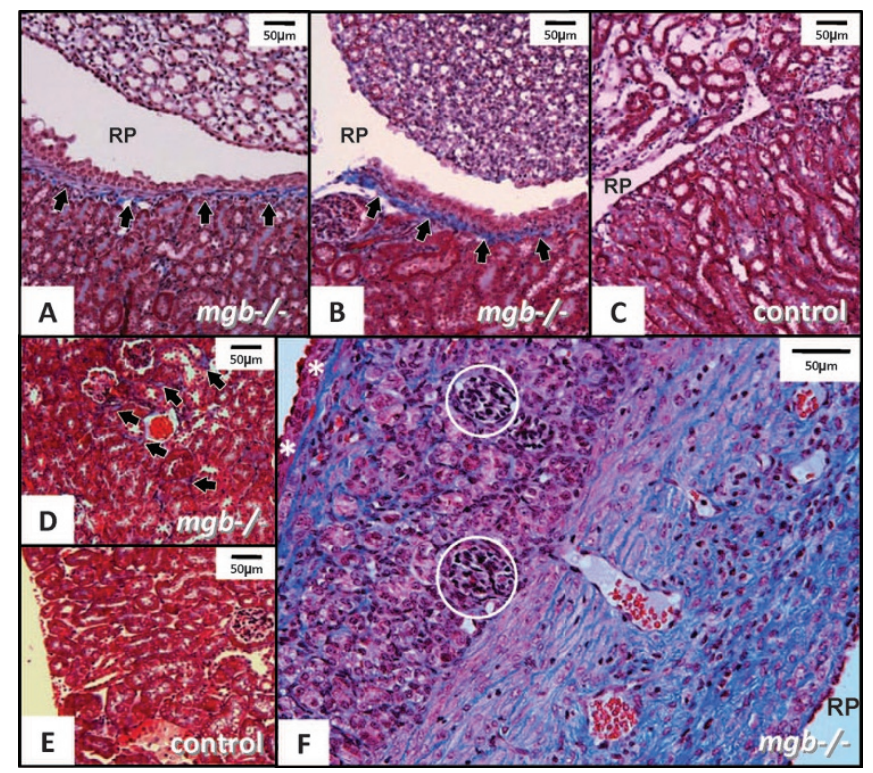

Figure 5. Trichrome-staining in AD kidneys. A, Moderate $m g b-/-$ kidney showing fibrotic band underlying urothelium of renal pelvis $(R P$, arrows). $B$, Nonhydronephrotic contralateral $m g b-/-$ kidney of same mouse showing similar fibrotic band underlying the urothelium (arrows). C, Control kidney showing no fibrotic band. $D$, Outer renal cortex in moderate $m g b-/-$ kidney with interstitial collagen accumulation (arrows) vs control (E). A-E, $\times 10$. F, Severe $m g b-/-$ kidney showing marked collagen deposition throughout compressed parenchyma. Glomeruli (circles) appear compressed, and bands of fibrosis are evident beneath the renal capsule (*) and throughout medulla, $\times 20$.

renal pelvis, and medullary tubules where an apical pattern of expression was observed (Fig. $8 G-I$ ). TGF- $\beta 1$ staining in severe kidneys was also observed in the surrounding regions of necrosis and within areas containing inflammatory infiltrates (data not shown). Expression of CTGF, a downstream target of TGF- $\beta 1$, was observed in the renal pelvis urothelium and collecting duct epithelia in moderate to severe AD kidneys but not controls (Fig. 9). 


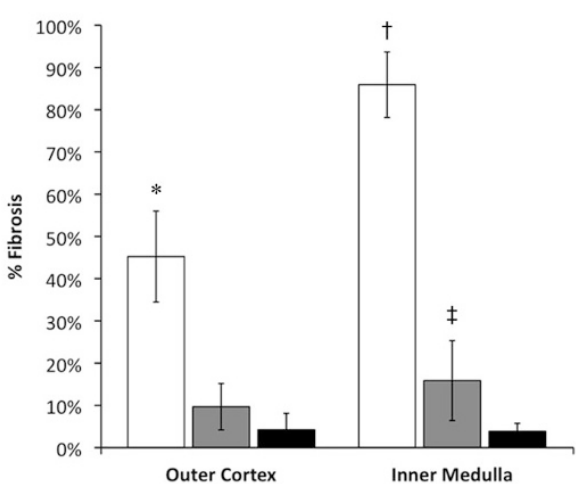

Figure 6. Quantitation of fibrosis. AD kidneys were stratified into severe $(\square)$ or moderate $(\square)$ groups as graded by ultrasound. Fibrosis was scored in multiple fields for each kidney, and the mean scores were compared with controls (ם). ${ }^{*} p=9 \times 10^{-7} ; \dagger p=4 \times 10^{-8}$; and $\ddagger p=0.04$.

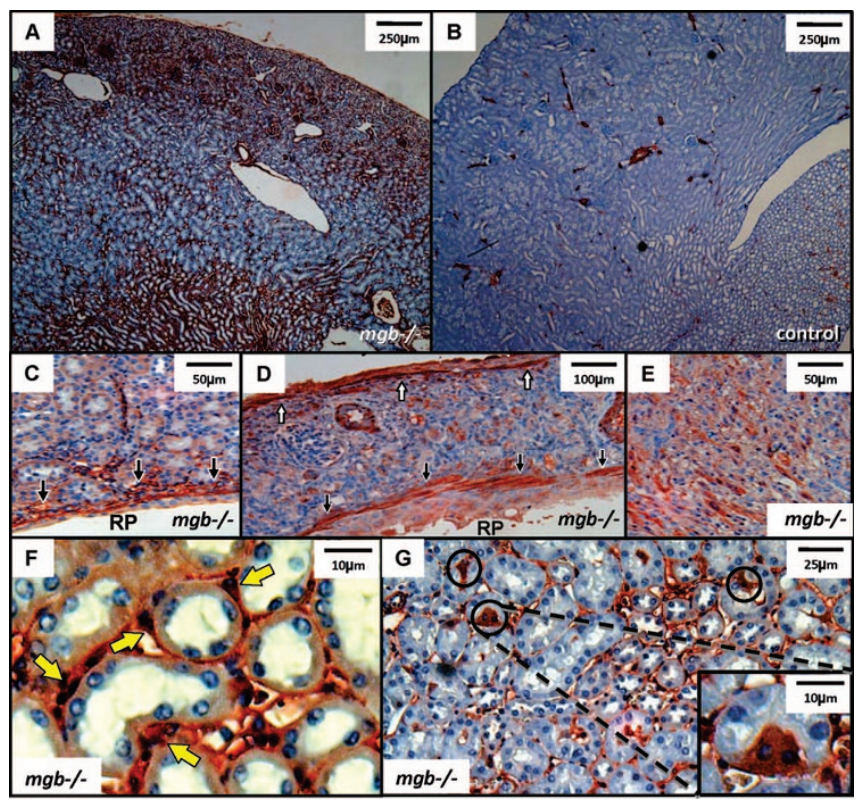

Figure 7. $\alpha$-SMA expression in AD kidneys. $A$, Moderate $m g b-/-$ kidney showing expanded $\alpha$-SMA expression $v s$ control $(B), \times 4 . C, \alpha$-SMA expression underlying renal pelvis $(R P)$ urothelium in moderate $m g b-/-$ kidneys, $\times 20$. $D, \alpha$-SMA staining in subcapsular region (white arrows) and underlying renal pelvis urothelium (black arrows) in severe $m g b-/-$ kidney, $\times 10$. E, Widespread $\alpha$-SMA-positive cells in interstitium of severe $m g b-/-$ kidney, $\times 20$. $F, \alpha$-SMA-positive cells form smooth muscle collars (yellow arrows) in severe $m g b-/-$ kidneys, $\times 100$. $G$, Scattered tubular epithelial cells express $\alpha$-SMA (circles) in severe $m g b-/-$ kidneys, $\times 40$, inset $\times 100$.

In severe AD kidneys, scattered renal tubular cells showed a loss of polarized E-cadherin localization (Fig. 10). Severe kidneys also showed increased E-cadherin expression throughout the urothelium of the renal pelvis that was not observed in controls (data not shown).

$\mathrm{F} 4 / 80$ expression was almost undetectable in AD control kidneys (Fig. 11A). In contrast, F4/80 expression in presumptive macrophages progressively increased in moderate to severe $\mathrm{AD}$ kidneys with positively staining cells observed throughout the interstitium and glomeruli (Fig. 11B-D).

AD control kidneys showed sparse WT-1 positive cells within their glomeruli (Fig. 12A), whereas the glomeruli of severe kidneys showed a statistically significant increase in

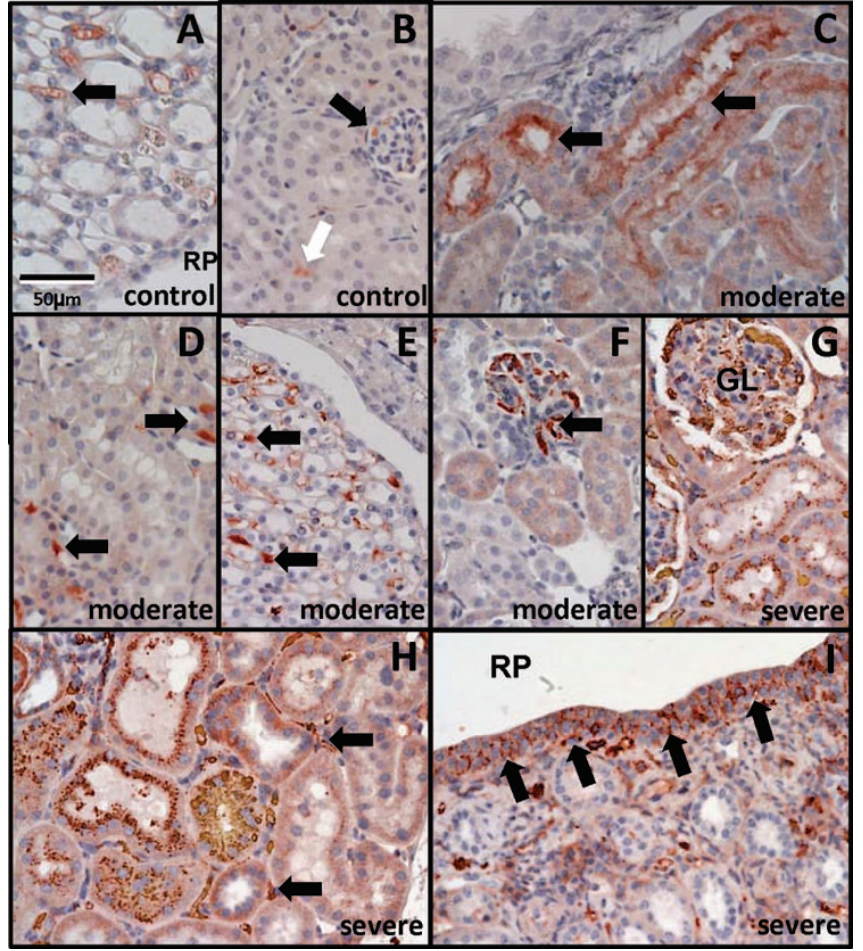

Figure 8. TGF $\beta 1$ expression in AD kidneys. Control kidneys show TGF $\beta 1$ staining in interstitium of renal papilla $(A$, arrow) and cortex $(B$, white arrow), and glomeruli $(B$, black arrow). Moderate $m g b-/-$ kidneys show expanded TGF $\beta 1$ staining within medullary tubules $(C$, arrows), cortical and medullary interstitial cells ( $D$ and $E$, arrows), and glomeruli $(F$, arrow). Severe $m g b-/-$ kidneys show expanded TGF $\beta 1$ staining in cortical tubules $(G)$, glomeruli $(G$, $G L)$, medulla $(H)$, interstitial cells $(H$, arrows), and renal pelvis $(R P)$ urothelium $(I$, arrows $), \times 40$.

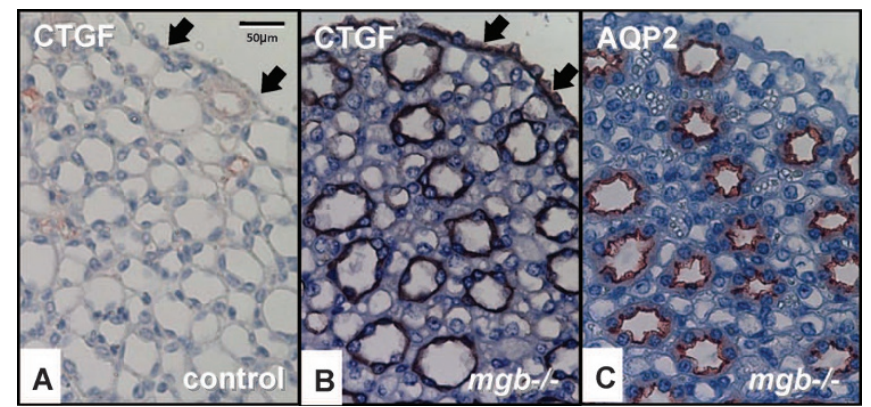

Figure 9. CTGF expression in AD kidneys. CTGF expression in the urothelium (arrows) and collecting ducts of control $(A)$ and severe $m g b-/-(B)$ kidneys. $C, \mathrm{AQP} 2$ staining of adjacent serial section to $B, \times 40$.

WT-1 positive cells with a distribution similar to that observed in NB control glomeruli (Fig. 12B-D).

Pax2 staining in $\mathrm{AD}$ control kidneys was reduced when compared with NB control kidneys. NB control kidneys showed intense nuclear and diffused cytoplasmic staining throughout the nephrogenic zone, in a subset of cortical and medullary tubules, and in the urothelium lining the renal pelvis (Fig. 13A-D). Pax2 staining in severe AD $m g b-/-$ kidneys was heterogeneous, with some animals displaying high levels of Pax2 staining throughout the renal parenchyma and the urothelium of the renal pelvis similar to that observed in NB kidneys (Fig. 13E). AD control kidneys showed limited Pax2 staining in the cells lining Bowman's capsule (Fig. 13F), 


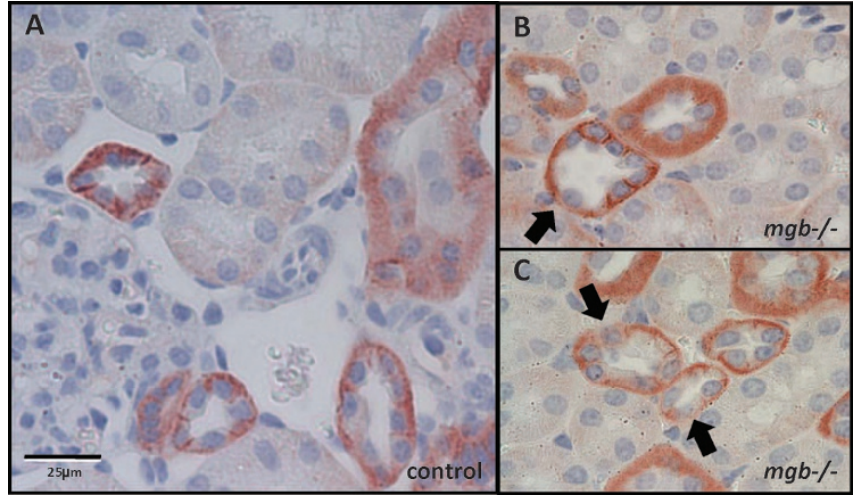

Figure 10. E-cadherin expression in $\mathrm{AD}$ kidneys. E-cadherin immunohistochemistry in control $(A)$ and severe $m g b-/-(B$ and $C)$ renal cortex, $\times 40$. Arrows indicate redistribution of E-cadherin in scattered tubular cells in the mutant.

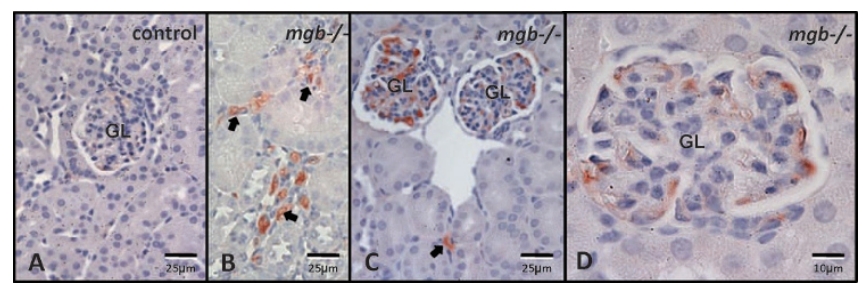

Figure 11. F4/80 expression in AD kidneys. (A) Control. (B-D) Severe $m g b-/-$ kidneys show expanded F4/80-positive cells within the interstitium (arrows) and glomeruli (GL). A-C, $\times 40 ; D, \times 100$.

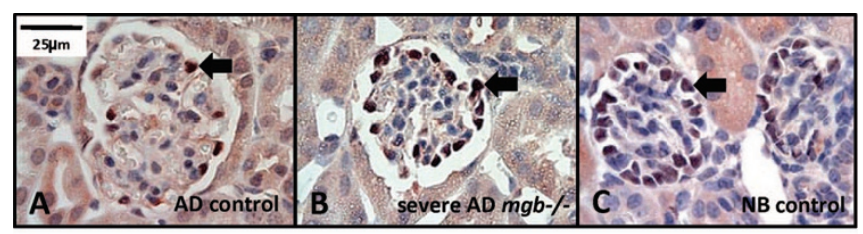

\section{D}

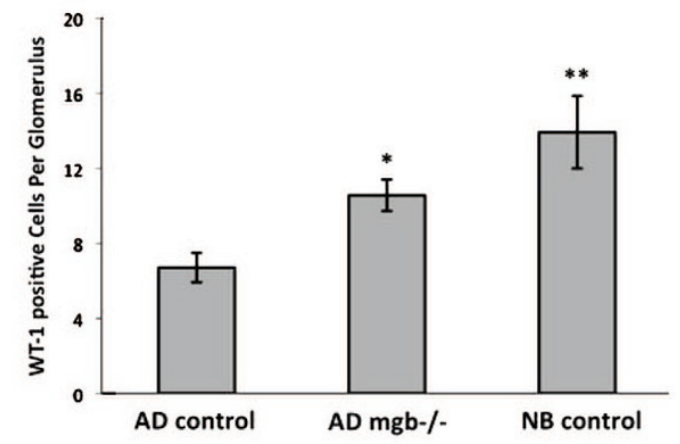

Figure 12. WT-1 expression in $\mathrm{NB}$ and $\mathrm{AD}$ kidneys. $A, \mathrm{AD}$ control glomeruli show fewer WT-1 positive cells than glomeruli from severe AD $m g b-1-$ kidneys $(B)$, which appear more similar to NB control glomeruli $(C), \times 100$. $D$, Frequency of WT-1 positive cells in AD control, AD $m g b-/-$, and NB control glomeruli. $* p=9 \times 10^{-6}$ and $* * p=5 \times 10^{-4}$.

whereas severe AD kidneys showed a statistically significant increase in the number of Pax2-positive cells lining Bowman's capsule to a level similar to that observed in NB controls (Fig. $13 G$ and $H$ ).

No significant difference in the extent or distribution of TUNEL-positive cells was observed between mutant and control kidneys at any of the time points examined (data not shown).

Rescue of mgb-/- mice. Cutaneous vesicostomy was performed on 19 male $m g b-/-$ mice (Table 3). Five mice
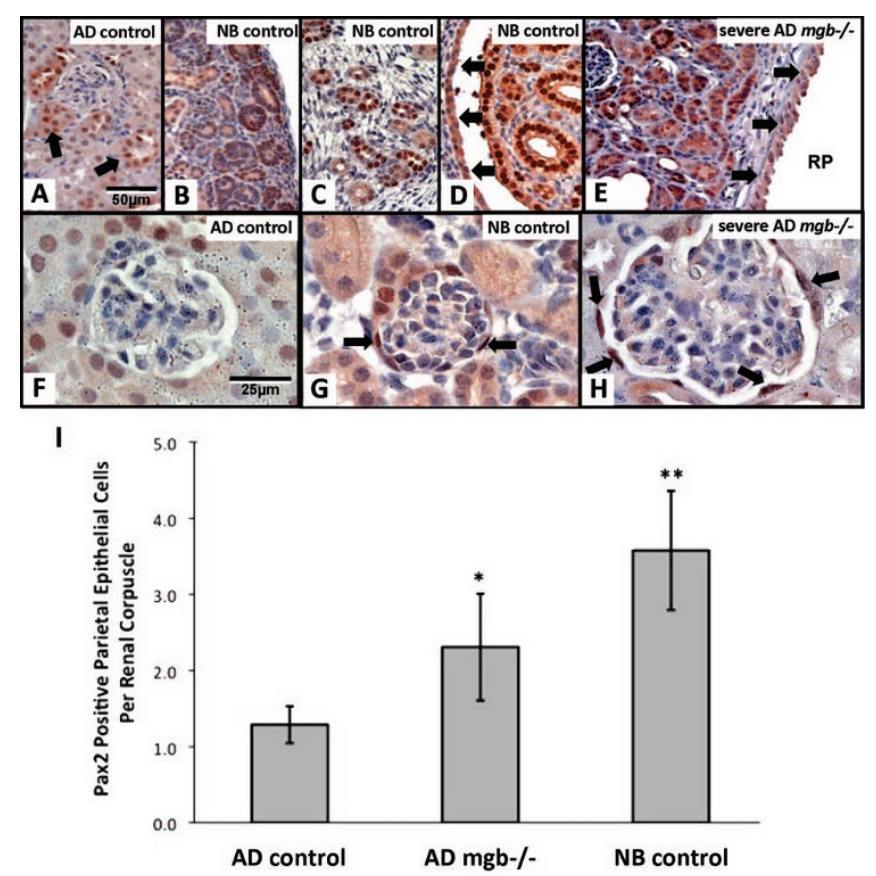

Figure 13. Pax 2 expression in $\mathrm{NB}$ and $\mathrm{AD}$ kidneys. $A, \mathrm{AD}$ control kidney shows minimal Pax2 staining in scattered tubules (arrows). NB control kidneys show Pax2 staining in immature nephrons of the cortex $(B)$ and medulla $(C)$, renal papillae $(D)$, and renal pelvis urothelium $(D$, arrows). $E$, Severe AD $m g b-/-$ kidneys show expanded Pax2 staining in compressed parenchyma and renal pelvis urothelium (RP, arrows) reminiscent of $\mathrm{NB}$ control kidneys. $A-E, \times 40$. Pax2 staining of glomeruli in AD control $(F), \mathrm{NB}$ control $(G)$, and severe AD $m g b-/-(H)$ kidneys. Severe AD $m g b-/-$ kidneys $(H)$ show Pax2-positive cells within Bowman's capsule similar to NB controls $(G) . F-H, \times 100$. I, Frequency of Pax 2 positive cells in AD control, $\mathrm{AD} m g b-/-$, and NB control renal corpuscles. ${ }^{*} p=0.03$ and ${ }^{* *} p=0.004$.

Table 3. Outcomes after cutaneous vesicostomy in $\mathrm{mgb}-1-$ male mice

\begin{tabular}{lcl}
\hline \multicolumn{3}{c}{ Age at } \\
Animal & surgery $(\mathrm{d})$ & \multicolumn{1}{c}{ Status/cause of death } \\
\hline 1869 & 32 & Lived several mo and bred well \\
1923 & 39 & Lived several mo and bred well \\
2021 & 42 & Lived for about 3 mo \\
2309 & 31 & Lived for about 3 mo (did not breed well) \\
2264 & 28 & Killed at 14 wk of age but looked well \\
2409 & 27 & Alive, stoma patent, and thriving at 15 weeks of age \\
1986 & 19 & Apparent renal failure \\
1987 & 19 & Apparent renal failure \\
1988 & 16 & Apparent renal failure \\
1989 & 16 & Apparent renal failure \\
1999 & 26 & Apparent renal failure \\
2298 & 59 & Apparent renal failure \\
2299 & 60 & Apparent renal failure \\
2419 & 30 & Apparent renal failure \\
1933 & 63 & Medical demise (morphine overdose) \\
1998 & 33 & Surgical complication (wound dehiscence) \\
2008 & 28 & Surgical complication (bladder prolapse) \\
2348 & 27 & Surgical complication (uroperitoneum) \\
2349 & 27 & Surgical complication (bladder prolapse) \\
\hline
\end{tabular}

(26\%) died from intraoperative or perioperative complications. Of the mice that survived, eight animals (42\%) died between 1 and 2 wk postoperatively. Examination of each animal revealed a patent stoma, well-healed abdominal wall, and no evidence of organ prolapse. Six mice (32\%) that 
underwent vesicostomy survived an average of $17.8 \mathrm{wk}$ and were considered surgical and therapeutic successes, because $99 \%$ of male $m g b-/-$ mice die by 6 wk of age (3). Two of the surviving animals became successful breeders.

\section{DISCUSSION}

Genetic models of obstructive nephropathy have been previously described (4-7). Postnatal models of obstruction include congenital progressive hydronephrosis and targeted deletions in a disintegrin and metalloproteinase with thrombospondin motifs-1, lysosomal membrane protein LIMP-2/LGP85, and calcineurin. Transgenic mice overexpressing human chorionic gonadotropin develop functional urethral obstruction in adulthood. Genetic models that develop in utero obstruction include some lines of Wistar rats, mice heterozygous for bone morphogenetic protein 4, and mice deficient in the transcription factor Id 2 and angiotensin type 2-receptor. Unfortunately, all these models possess complicated phenotypes including renal hypoplasia, dysplasia, aplasia, megaureters, and duplicated ureters that confound their utility as simple models of CON. None of the defects observed in these animals is similar to the $m g b$ phenotype, making it a unique genetic model for the study of CON.

The preponderance of data on progressive renal injury during obstruction comes from surgical ureteral ligation in neonatal and $\mathrm{AD}$ rats, mice, and pigs (8). Studies using unilateral ureteral obstruction (UUO) suggest that progressive renal injury involves four overlapping stages comprising interstitial inflammation, tubular and myofibroblast proliferation, tubular apoptosis, and interstitial fibrosis. The precise physiological relevance of these postnatal models to the development of $\mathrm{CON}$ remains to be elucidated.

Pathogenic changes in kidney development are first detected in $m g b-/-$ mice at birth, suggesting that their kidneys possess preexisting pathological changes resulting from in utero obstruction. These changes may not be as severe as those observed in some patients and large animal models of in utero obstruction. This fact most likely reflects differences in rodent renal development, because postnatal murine kidney maturation correlates to kidney development in the third trimester in humans (9). This observation greatly extends the utility of the $m g b-/-$ mouse model because this important postnatal phase of murine kidney development occurs in the face of progressively worsening hydronephrosis in a manner similar to that observed during late gestation in human CON.

The earliest histopathological change detected in NB $m g b-/-$ kidneys was expanded $\alpha$-SMA expression throughout the cortex and medulla. Changes in $\alpha$-SMA-positive staining have been correlated with the appearance of activated myofibroblasts in a variety of pathological settings $(10,11)$. Renal myofibroblasts may be derived from multiple cellular lineages including hematopoietic progenitor cells, pericytes, preexisting interstitial myofibroblasts, and epithelial-tomesenchymal transition (EMT) (12). Although previous studies highlight the importance of EMT in the development of renal fibrosis $(13,14)$, the initial appearance of $\alpha$-SMApositive myofibroblasts in NB $m g b-/-$ kidneys occurs before any obvious changes in EMT, TGF- $\beta 1$ expression, or renal fibrosis. This suggests that these early activated myofibroblasts might be derived from one of the alternative lineages mentioned above and is consistent with recent studies suggesting that EMT may not be the primary source of activated myofibroblasts during kidney pathogenesis (15). The appearance of these myofibroblasts at this early stage is unique to the $m g b$ mouse model and may be critical in priming the renal interstitium for subsequent fibrotic changes.

As the duration and degree of hydronephrosis progressively worsens in AD $m g b-/-$ mice, renal fibrosis becomes apparent. Fibrosis initiates under the urothelium of the renal pelvis, secondarily appears beneath the renal capsule, and finally spreads through the interstitium as the condition worsens. The initial fibrotic changes underlying the renal pelvis and capsule may represent structural responses to progressively worsening hydronephrosis. Both changes would be predicted to reinforce the corresponding epithelial membranes, thereby preventing rupture and subsequent extravasation of urine into the kidney parenchyma or peritoneum. Secondary development of interstitial fibrosis provides collagen to preserve the structural integrity of the organ, whereas at the same time generating an expanded myofibroblast population necessary for eventual repair (14). Fibrotic changes in the contralateral kidney of affected mice in the absence of discernable hydronephrosis suggest the presence of a humoral or mechanical factor or both that "primes" the contralateral kidney before the development of pelvicaliectasis. The progressive pattern of fibrotic development seems unique to the $m g b$ mouse and most likely reflects the gradual development of hydronephrosis observed in these animals versus other more acute obstructive models.

The distribution of $\alpha$-SMA expression in severe kidneys closely mirrors the fibrotic pattern described above. This observation supports previous work implicating this cell type in the production of renal fibrosis (10). Alterations in intracellular E-cadherin and $\alpha$-SMA distribution in scattered tubule cells of AD $m g b-/-$ mice suggest that EMT occurs in response to the development of interstitial fibrosis. Similar observations have been made in surgical models and human studies of obstructive nephropathy, suggesting that EMT of tubular epithelial cells is a key source of activated interstitial myofibroblasts (11). It seems plausible that at this later stage of pathogenesis, one of the primary sources for the continued expansion of interstitial myofibroblasts may be activated EMT of tubular epithelial cells. These results suggest that activated myofibroblasts derive from multiple sources in $m g b-/-$ mice based on the stage of disease progression. Future studies will examine both the phenotype and origin of this important cell type in $m g b-/-$ mice.

AD $m g b-/-$ kidneys showed expanded TGF- $\beta 1$ and CTGF expression in a pattern reminiscent of that observed for collagen deposition and $\alpha$-SMA expression. These observations are similar to that reported in fibrotic kidneys after UUO (16). TGF- $\beta 1$ mediates development of renal fibrosis through the activation of local myofibroblasts and production of CTGF, $\beta$-catenin, angiotensin II, and hepatocyte growth factor (17-20). In addition, TGF- $\beta 1$ is known to promote tubular epithelial cell transdifferentiation to myofibroblasts, thereby 
modulating EMT $(18,21)$. CTGF induction by TGF- $\beta 1$ is postulated to mediate its profibrotic activity by modulating fibroblast growth and ECM production (16), whereas the stimulation of renal tubular epithelial cells with TGF- $\beta 1$ increases both CTGF and $\alpha$-SMA transcription (22). This suggests that the fibrotic changes observed in $m g b-/-$ kidneys develop by modulating the TGF- $\beta 1$ signaling pathway, the differentiation of interstitial myofibroblasts, and subsequent collagen deposition in a manner similar to that reported in previous models of obstruction.

Inflammatory cells first become evident in severe $\mathrm{AD}$ $m g b-/-$ kidneys. This observation is in contrast to previous studies where interstitial inflammation represents an early response to UUO (23) and suggests that this may not be the case in CON. However, the presence of lymphocytes/ monocytes, plasma cells, and macrophages in severe kidneys during the interstitial expansion of myofibroblasts suggests that these cells may play an important role during this later phase of pathogenesis.

Although previous models of obstruction have suggested a significant role for tubular apoptosis in regulating the renal response to urinary tract obstruction, we did not observe a significant increase in apoptosis in the affected kidneys of $m g b-/-$ mice. This observation is similar to other genetic models of CON and suggests that tubular apoptosis may play a limited role in CON (23). Severe $m g b-/-$ kidneys did show significant regions of necrosis that were often associated with surrounding inflammatory cells. It is plausible, therefore, that this necrotic inflammatory response contributes to cell death independent of cellular apoptosis in $m g b-/-$ kidneys.

Severe $m g b-/-$ kidneys possessed several dysplastic features including loss of normal kidney tissue organization, cyst formation, and development of peritubular smooth muscle collars. In addition, the distribution of WT-1 and Pax2 staining observed in severe kidneys was suggestive of an immature glomerular phenotype in AD $m g b-/-$ mice. The persistence or reactivation of WT-1 or Pax 2 expression has been implicated in many forms of renal pathology including UUO and cystic kidney disease (24-26). These observations are consistent with classic work by Potter (27) suggesting that the renal pathology associated with congenital anomalies of the kidney and urinary tract includes varying degrees of renal dysplasia.

Although the variable disease progression observed in $m g b-/-$ mice seems similar to that observed in humans, it initially complicated the pathophysiological analysis of these animals. The use of small animal ultrasound resolved this issue by permitting easy stratification of $m g b-/-$ mice into consistent pathological study groups composed of mild, moderate, and severe kidneys. This approach significantly improves the utility of this animal model. Interestingly, this analysis also indicated that $m g b-/-$ mice preferentially develop unilateral, right-sided hydronephrosis. This observation is similar to the "pop off" mechanism proposed in children with secondary unilateral vesicoureteral reflux, which has been suggested to be protective of renal function in the contralateral kidney by reducing intravesical pressure (28).

Our previous work indicates that $m g b-/-$ mice die from the complications of renal failure (3). The surgical rescue of male $m g b-/-$ mice via vesicostomy confirmed these initial observations by relieving the obstruction, thereby sparing kidney function and extending life. Interestingly, of the $74 \%$ of animals that survived the perioperative period, $\sim 40 \%$ died within the first 2 wk although they possessed a patent stoma. This observation is reminiscent of the fact that $30-50 \%$ of the children presenting with posterior urethral valves develop chronic kidney insufficiency or end-stage renal disease despite clinical intervention (29).

In summary, this study demonstrates that $m g b-/-$ mice develop progressive renal injury that is directly related to the severity of hydronephrosis present in the animal. The timing and pattern of renal pathogenesis observed in $m g b-/-$ kidneys seems unique from any previous genetic or surgical models of obstruction and suggests an initial adaptive response followed by a progressive loss of tissue integrity. In addition, many of the pathophysiological traits observed in the $m g b$ mouse model seem similar to those observed in children with CON. These observations indicate that the $m g b$ mouse is a useful model to study the development, progression, and pathophysiology of CON. In conclusion, the $m g b$ mouse represents a unique small animal model of CON and affords us the opportunity to identify and manipulate the key molecular pathways associated with the development of chronic renal failure, whereas at the same time providing a platform for biomarker development and the assessment of surgical and therapeutic strategies designed to ameliorate the pathophysiological effects of kidney disease in these animals.

\section{REFERENCES}

1. North American Pediatric Renal Transplant Cooperative Study 2008 annual report Available at: https://web.emmes.com/study/ped/annlrept/annlrept.html. Accessed March 10, 2010

2. Roth KS, Carter WH Jr, Chan JC 2001 Obstructive nephropathy in children: long-term progression after relief of posterior urethral valve. Pediatrics 107:10041010

3. Singh S, Robinson M, Nahi F, Coley B, Robinson ML, Bates CM, Kornacker K, McHugh KM 2007 Identification of a unique transgenic mouse line that develops megabladder, obstructive uropathy, and renal dysfunction. J Am Soc Nephrol 18:461-471

4. Chevalier RL, Peters CA 2009 Obstructive uropathy. In; Avner E, Harmon W, Niaudet P, Yoshikawa N (eds) Pediatric Nephrology. Springer, Berlin, Heidelberg, pp 1337-1377

5. Miller J, Hesse M, Diemer T, Haenze J, Knerr I, Rascher W, Weidner W 2004 Congenital unilateral ureteropelvic junction obstruction of the rat: a useful animal model for human ureteropelvic junction obstruction? Urology 63:190-194

6. Rulli SB, Ahtiainen P, Makela S, Toppari J, Poutanen M, Huhtaniemi I 2003 Elevated steroidogenesis, defective reproductive organs, and infertility in transgenic male mice overexpressing human chorionic gonadotropin. Endocrinology 144:4980-4990

7. Weide LG, Lacy PE 1991 Hereditary hydronephrosis in C57BL/KsJ mice. Lab Anim Sci 41:415-418

8. Chevalier RL, Forbes MS, Thornhill BA 2009 Ureteral obstruction as a model of renal interstitial fibrosis and obstructive nephropathy. Kidney Int 75:11451152

9. Moritz KM, Wintour-Coghlan M, Black MJ, Bertram JF, Caruana G 2008 Factors Influencing Mammalian Kidney Development: Implications for Health in Adult Life. Springer-Verlag, Berlin, Germany, pp 1-9

10. Grande MT, López-Novoa JM 2009 Fibroblast activation and myofibroblast generation in obstructive nephropathy. Nat Rev Nephrol 5:319-328

11. Hinz B 2007 Formation and function of the myofibroblast during tissue repair J Invest Dermatol 127:526-537

12. Bascands JL, Schanstra JP 2005 Obstructive nephropathy: insights from genetically engineered animals. Kidney Int 68:925-937

13. Butt MJ, Tarantal AF, Jimenez DF, Matsell DG 2007 Collecting duct epithelialmesenchymal transition in fetal urinary tract obstruction. Kidney Int 72:936944

14. Iwano M, Plieth D, Danoff TM, Xue C, Okada H, Neilson EG 2002 Evidence that fibroblasts derive from epithelium during tissue fibrosis. J Clin Invest 110:341-350 
15. Picard N, Baum O, Vogetseder A, Kaissling B, Le Hir M 2008 Origin of renal myofibroblasts in the model of unilateral ureter obstruction in the rat. Histochem Cell Biol 130:141-155

16. Okada H, Kikuta T, Kobayashi T, Inoue T, Kanno Y, Takigawa M, Sugaya T, Kopp JB, Suzuki H 2005 Connective tissue growth factor expressed in tubular epithelium plays a pivotal role in renal fibrogenesis. J Am Soc Nephrol 16:133-143

17. Böttinger EP, Bitzer M 2002 TGF-beta signaling in renal disease. J Am Soc Nephrol $13: 2600-2610$

18. Liu Y 2010 New insights into epithelial-mesenchymal transition in kidney fibrosis. J Am Soc Nephrol 21:212-222

19. Shi-Wen X, Leask A, Abraham D 2008 Regulation and function of connective tissue growth factor/CCN2 in tissue repair, scarring and fibrosis. Cytokine Growth Factor Rev 19:133-144

20. Yang J, Dai C, Liu Y 2005 A novel mechanism by which hepatocyte growth factor blocks tubular epithelial to mesenchymal transition. J Am Soc Nephrol 16:68-78

21. Border WA, Noble NA 1997 TGF-beta in kidney fibrosis: a target for gene therapy. Kidney Int 51:1388-1396

22. Zhang C, Meng X, Zhu Z, Yang X, Deng A 2004 Role of connective tissue growth factor in renal tubular epithelial-myofibroblast transdifferentiation and extracellular matrix accumulation in vitro. Life Sci 75:367-379
23. Chevalier RL 2006 Pathogenesis of renal injury in obstructive uropathy. Curr Opin Pediatr 18:153-160

24. Cohen T, Loutochin O, Amin M, Capolicchio JP, Goodyer P, Jednak R 2007 PAX2 is reactivated in urinary tract obstruction and partially protects collecting duct cells from programmed cell death. Am J Physiol Renal Physiol 292:F1267F1273

25. Dressler GR, Woolf AS 1999 Pax2 in development and renal disease. Int J Dev Biol 43:463-468

26. Pritchard-Jones K, Fleming S, Davidson D, Bickmore W, Porteous D, Gosden C, Bard J, Buckler A, Pelletier J, Housman D, van Heyningen V, Hastie N 1990 The candidate Wilms' tumour gene is involved in genitourinary development. Nature 346:194-197

27. Potter EL 1972 Normal and Abnormal Development of the Kidney. Year Book Medical Publishers, Inc., Chicago, IL

28. Greenfield SP, Hensle TW, Berdon WE, Wigger HJ 1983 Unilateral vesicoureteral reflux and unilateral nonfunctioning kidney associated with posterior urethral valves-a syndrome? J Urol 130:733-738

29. Hutton KA 1994 Posterior urethral valves. Br J Urol 74:134 